\title{
HAD hydrolase function unveiled by substrate screening: enzymatic characterization of Arabidopsis thaliana subclass I phosphosugar phosphatase AtSgpp
}

\author{
José A. Caparrós-Martín · Iva McCarthy-Suárez • \\ Francisco A. Culiáñez-Macià
}

Received: 28 June 2012/ Accepted: 2 November 2012/Published online: 24 November 2012

(C) The Author(s) 2012. This article is published with open access at Springerlink.com

\begin{abstract}
This work presents the isolation and the biochemical characterization of the Arabidopsis thaliana gene AtSgpp. This gene shows homology with the Arabidopsis low molecular weight phosphatases AtGpp1 and AtGpp2 and the yeast counterpart GPP1 and GPP2, which have a high specificity for DL-glycerol-3-phosphate. In addition, it exhibits homology with DOG1 and DOG2 that dephosphorylate 2-deoxy-D-glucose-6-phosphate. Using a comparative genomic approach, we identified the AtSgpp gene as a conceptual translated haloacid dehalogenase-like hydrolase HAD protein. AtSgpp (locus tag At2g38740), encodes a protein with a predicted $\mathrm{Mw}$ of $26.7 \mathrm{kDa}$ and a $\mathrm{pI}$ of 4.6. Its sequence motifs and expected structure revealed that AtSgpp belongs to the HAD hydrolases subfamily I, with the C1-type cap domain. In the presence of $\mathrm{Mg}^{2+}$ ions, the enzyme has a phosphatase activity over a wide range of phosphosugars substrates ( $\mathrm{pH}$ optima at 7.0 and $K_{\mathrm{m}}$ in the range of 3.6-7.7 mM). AtSgpp promiscuity is preferentially detectable on D-ribose-5-phosphate, 2-deoxy-D-ribose5-phosphate, 2-deoxy-D-glucose-6-phosphate, D-mannose6-phosphate, D-fructose-1-phosphate, D-glucose-6-phosphate, DL-glycerol-3-phosphate, and D-fructose-6-phosphate, as substrates. AtSgpp is ubiquitously expressed throughout development in most plant organs, mainly in sepal and guard cell. Interestingly, expression is affected by abiotic and biotic stresses, being the greatest under Pi starvation and cyclopentenone oxylipins induction. Based on both,
\end{abstract}

J. A. Caparrós-Martín · I. McCarthy-Suárez ·

F. A. Culiáñez-Macià ( $\square)$

Instituto de Biología Molecular y Celular de Plantas "Eduardo

Primo Yúfera" (UPV-CSIC), Universidad Politécnica de

Valencia, Ciudad Politécnica de la Innovación (CPI),

C/Ingeniero Fausto Elio s/n, 46022 Valencia, Spain

e-mail: faculia@ibmcp.upv.es substrate lax specificity and gene expression, the physiological function of AtSgpp in housekeeping detoxification, modulation of sugar-phosphate balance and Pi homeostasis, is provisionally assigned.

Keywords Abiotic and biotic stress - Arabidopsis . HAD superfamily · Hydrolases · Pi homeostasis . Sugar phosphatases

\begin{abstract}
Abbreviations
HAD Haloacid dehalogenase-like hydrolase proteins MBP Maltose-binding protein
\end{abstract}

\section{Introduction}

The haloacid dehalogenase-like hydrolase (HAD) superfamily is a large group of proteins with diverse substrate specificity whose members, despite the family name, are involved not only in the enzymatic cleavage by nucleophilic substitution of carbon-halogen bonds (C-halogen), but also in a variety of hydrolytic enzyme activities including phosphatase (CO-P), phosphonatase (C-P) and phosphoglucomutase (CO-P hydrolysis and intramolecular phosphoryl transfer) reactions (Koonin and Tatusov 1994; Allen and Dunaway-Mariano 2004).

Within the HAD superfamily, the wide family of magnesium-dependent acid phosphatases and phosphomutases is characterized by an amino-terminal conserved Asp as the nucleophile (Collet et al. 1998; Selengut 2001). All members share the $\alpha / \beta$ core domain catalytic scaffold, with the active site formed by four loops containing highly conserved sequence motifs (loops 1, DxD; 2, T/S; 3, K/R; 4, $\mathrm{E} / \mathrm{DD}$, GDxxxD, or GDxxxxD), spatially arranged around a 
single binding cleft, that position substrate-cofactor binding and catalytic residues that are involved in the core chemistry (Burroughs et al. 2006). Many of them also possess a smaller cap domain, linked to the core by two hinge-like solvated peptide linkers, which acts as a dynamic rigid lid over the core active site. Although its primary function might be active-site desolvation, the cap domain contains the helix-loop-helix (loop 5) with a stringently conserved Gly flanked by residues whose side chains contribute to the catalytic site formed at the domain-domain interface. These residues are responsible for the chemistry diversification within the family and provide substrate specificity (Allen and Dunaway-Mariano 2004; Lahiri et al. 2004). Thus, the elements involved in substrate-recognition and chemical-catalysis are located separately; substrate recognition is delegated to cap residues, whereas phosphoryl transfer is mediated by residues located deep inside the core cleft (Wang et al. 2002; Lu et al. 2005, 2008).

Based on the presence, topology and location of the cap domain, the HAD superfamily is divided into three subfamilies (I, IIA-IIB and III) (Morais et al. 2000; Zhang et al. 2002; Tremblay et al. 2006). In subfamily I, the small $\alpha$-helical-bundle cap is inserted between loops 1 and 2 in the core domain, whereas in subfamily II the larger $\beta$-sandwich is placed between loops 2 and 3; the third group contains no insertion (Selengut and Levine 2000; Shin et al. 2003). Unlike the core catalytic domain, the cap has undergone extensive evolutionary diversity in substrate exploration during HADs evolution (Burroughs et al. 2006). While the $\alpha / \beta$ Rossmann core active sites are superimposable, the architecture of the cap domain differs even between the closest structural homologs (Rao et al. 2006).

In HAD phosphatases, after the binding of the substrate the active site closes and $\mathrm{Mg}^{2+}$ cofactor interacts with the negatively charged phosphate, preparing it for nucleophilic attack by the first conserved Asp, forming an intermediate acyl-phosphate with the carboxyl group. Then, the enzyme opens again allowing the escape of the leaving group and the entry of solvent; water is deprotonated by the second Asp, hydrolyzing the acyl-phosphate intermediate and returning the enzyme to the native status (Burroughs et al. 2006). In phosphohydrolases, the Asp nucleophile is located on loop 1, loop 2 positions a conserved Ser/Thr that binds the substrate phosphoryl group, whereas loop 3 positions a conserved Arg/Lys that orients and shields charge in the Asp nucleophile and the phosphoryl group and, finally, loop 4 positions two or three Asp residues that bind the $\mathrm{Mg}^{2+}$ cofactor (Lu et al. 2005).

Due to their sequence divergence, only the highly conserved catalytic motifs and the similar folds and active-site structures allow identification between members of the HADs (Morais et al. 2000). However, the catalyzed reaction and substrate specificity are difficult to predict and have to be determined empirically (Kuznetsova et al. 2006).

In an earlier work, we cloned and characterized two Arabidopsis thaliana isoforms AtGpp1 (At4g25840) and AtGpp2 (At5g57440) of the DL-glycerol-3-phosphatase, involved in plant glycerol metabolism (Caparrós-Martín et al. 2007). The analysis of the sequence indicates that AtGpp1 and AtGpp2 phosphatase are members of the HAD haloacid dehalogenase hydrolase superfamily. AtGpp1 and AtGpp2 show high homology with the yeast phosphatases GPP1 and GPP2 (Norbeck et al. 1996), which have a high specificity for DL-glycerol-3-phosphate, as well as with DOG1 and DOG2 that dephosphorylate 2-deoxy-D-glucose-6-phosphate (Rández-Gil et al. 1995).

The Arabidopsis genome contains homologous loci, other than AtGpp1 and AtGpp2, with similar scores and general function predicted as phosphatase/phosphohexomutase (unknown At2g38740, putative At4g39970, catalytic/ hydrolase At3g48420 and catalytic/hydrolase/phosphoglycolate phosphatase At2g33255). Attention was focused on these HAD superfamily-encoded loci, sharing significant similarity, presuming a related sequence-based assignment of activity on targeting substrates with similar structural characteristics, in Arabidopsis.

Following a screening approach, the four purified proteins were tested for phosphatase activity against a set of sugar phosphoesters. Pi release was only detected in assays with the product of locus At2g38740 so-called AtSgpp. Structural prediction and the chemistry analysis reveal AtSgpp as a typical phosphomonoesterase of subclass I (C1-type cap). At odds with other subfamily I representatives such as phosphonatase, phosphoserine phosphatase, 2,3-diketo-Lphospho-5-thiomethylpentane phosphatase, 2-deoxy-D-glucose-6-phosphate phosphatase, and glycerolphosphate phosphatase, rather than being substrate specific, AtSgpp shows a broad-range sugar phosphate phosphatase activity. Curiously enough, similar specificity has been ascribed to the Bacteroides thetaiotaomicron BT4131 gene ( $\mathrm{Lu}$ et al. 2005). BT4131 enzyme is member of the haloalkanoate dehalogenase superfamily (subfamily IIB, C2B-type cap), with a tentatively assigned physiological function in chitin metabolism. The expected structure, specificity and kinetics of plant AtSgpp chemistry were analyzed and compared with those of bacterial BT4131.

\section{Materials and methods}

\section{Materials}

The materials used for cloning were: pMAL-c2X vector and Escherichia coli TB1 host for expression (New 
England Biolabs, Hitchin, Hertfordshire, UK), First Strand DNA synthesis kit for reverse transcriptase PCR (Roche Applied Science, Mannheim, Germany), REDTaq DNA polymerase (Sigma, St. Louis, MO, USA), oligonucleotides (Sigma-Genosys, Gillingham, Dorset, UK) and pBluescript SK+ vector (StrataGene, Kirkland, WA, USA). The RNA extraction was achieved with GenElute mammalian total RNA kit RTN70/TriReagent T9424 (Sigma, St. Louis, MO, USA). The pSBETa helper vector was constructed at the Max-Planck Institut (Köln, Germany) (Schenk et al. 1995) and the total RNA from siliqua was extracted as in Vicient and Delseny (1999).

Plant material, growth conditions and stress treatments

Arabidopsis thaliana ecotype Columbia (Lehle Seeds, Round Rock, TX, USA) was grown in the greenhouse at $25{ }^{\circ} \mathrm{C}$ for $8 \mathrm{~h}$ in the dark and $16 \mathrm{~h}$ in light. For seedling stress assays, wild-type (WT) and transgenic surface-sterilized Arabidopsis seeds were sown in Petri dishes containing $3 \mathrm{ml}$ MSS medium [MS (Murashige and Skoog 1962) M5524 (Sigma) + $1 \%$ Agargel $^{\mathrm{TM}}$ A-3301 (Sigma) $+3 \%$ sucrose (Merck, Darmstadt, Germany)], MSS $+100 \mathrm{mM}$ $\mathrm{NaCl}$ (Panreac, Barcelona, Spain) or $15 \mathrm{mM} \mathrm{LiCl}$ (Panreac) for salt stress, MSS $+200 \mathrm{mM}$ sorbitol (Sigma) for osmotic stress, MSS $+5 \mathrm{mM} \mathrm{H}_{2} \mathrm{O}_{2}$ (Panreac) or $1 \mu \mathrm{M}$ methyl viologen (Sigma) for oxidative stress. Seedlings were grown for 12 days at $25{ }^{\circ} \mathrm{C}$ under fluorescent light, $8 \mathrm{~h}$ dark and $16 \mathrm{~h}$ light.

Genome, sequence and structural analysis

Comparative genomics were performed using programs such as BLAST (Altschul et al. 1990) and data bank resources from the NCBI. Protein domain families were generated with the ProDom program from the Swiss-Pro and TrEMBL sequence databases (Corpet et al. 2000). CLUSTAL $\mathrm{W}$ was used for the progressive multiple sequence alignment (Higgins et al. 1994). GENEVESTIGATOR Arabidopsis microarray database was utilized for the expression analysis (Zimmermann et al. 2004). 3D models of the protein have been built using the ESyPred3D web server (Lambert et al. 2002).

\section{cDNA cloning}

Using peptide sequence motifs shared between the yeast DL-glycerol-3-phosphatases (Norbeck et al. 1996), virtual clones were isolated by BLAST (Altschul et al. 1990) search screening from the predicted conceptual translated proteins of the A. thaliana genomic library. The corresponding homologous genes were cloned by reverse PCR using leaf total RNA as template for the first strand cDNA synthesis together with an oligo(dT) primer and avian myeloblastosis virus reverse transcriptase. The first strand cDNA template was PCR amplified using REDTaq DNA polymerase and the $5^{\prime}$-forward and $3^{\prime}$-reverse gene-specific adapted primers $5^{\prime}$-CGAGGAATTCATGAATGGCTTCT CTGATCTTAATCC-3'/5'-CCGGGTCGACTTAAGACT TGTTATCAAGTTCTTCC-3'; 5'-CGAGGAATTCATGG CGGTTTCTTGCAACCACTCTGC-3'/5'CCGGGTCGAC TTAAGCTGCAGTGACTATTGTTTGAAGC- ${ }^{\prime} ; 5^{\prime}$-CGA GGAATTCATGGCCACTGTGAAAATCTCTCTTTCC3'/5'-CCGGGTCGACTTAACTAACGAACTGTTTCCGG AG-3'; and $5^{\prime}$-CGAGGAATTCATGGCGAATTTAACG ACGAACGC- $3^{\prime} / 5^{\prime}$-CCGGGTCGACCTACGGGTTCAGG TCGAAGTTCG-3' for At2g38740, At4g39970, At3g484 20 and At2g33255, respectively. The PCR products were cloned as 735, 956, 960 or 675 bp EcoRI/SalI fragment into the pBluescript SK + vector. Sequencing of the pBluescript SK + clones revealed that the sequence of the proteins is the same as that published in the Gen-Bank ${ }^{\mathrm{TM}}$. The cDNAs, containing the entire gene coding region, were subcloned as EcoRI/SalI fragments into the pMAL-c2X expression vector and transformed into the expression strain $E$. coli $\mathrm{TB} 1$ for recombinant protein production. The cloning site used in the pMAL-c2X polylinker (located downstream of the malE gene), adding vector-encoded residues Ile-Ser-Glu-Phe fused between the factor $\mathrm{Xa}$ cleavage site and the $\mathrm{NH}_{2}$-terminal methionine residue of the cloned proteins. To improve the expression of the eukaryotic genes in the E. coli system, E. coli TB1 cells were co-transformed with pMAL-c2X At2g38740, pMALc2X At4g39970, pMAL-c2X At3g48420 or pMAL-c2X At2g33255 and, in each case, the helper plasmid pSBETa. The positive co-transformed colonies were selected on $200 \mu \mathrm{g} / \mathrm{ml}$ ampicillin (Sigma) and $100 \mu \mathrm{g} / \mathrm{ml}$ kanamycin (Sigma).

Purification of recombinant proteins

Selected co-transformed E. coli strains, containing fusion plasmid pMAL-c2X At2g38740, pMAL-c2X At4g39970, pMAL-c2X At3g48420 or pMAL-c2X At2g33255 and, in each case, the helper pSBETa, were gown at $37{ }^{\circ} \mathrm{C}$ to $2 \times 10^{8}$ cells $/ \mathrm{ml}\left(A_{600} \sim 0.5\right)$ in 11 of rich broth + glucose and ampicillin + kanamycin $(10 \mathrm{~g}$ tryptone, $5 \mathrm{~g}$ yeast extract, $5 \mathrm{~g} \mathrm{NaCl}, 2 \mathrm{~g}$ glucose, autoclave; add sterile $200 \mu \mathrm{g} / \mathrm{ml}$ ampicillin and $100 \mu \mathrm{g} / \mathrm{ml}$ kanamycin), induced with $1 \mathrm{mM}$ isopropyl- $\beta$-D-thiogalactoside (IPTG) (Ambion, Austin, TX, USA) and harvested $2 \mathrm{~h}$ after induction. Fusion proteins were released from the harvested cells by sonication in column buffer $(20 \mathrm{mM}$ Tris- $\mathrm{HCl} \mathrm{pH} 7.4$, $200 \mathrm{mM} \mathrm{NaCl}$, and $1 \mathrm{mM}$ EDTA), collected after elution from the amylose resin (New England Biolabs) with 
column buffer $+10 \mathrm{mM}$ maltose (Sigma) and the concentration determined by the Bradford method (1976). Proteins were separated by SDS-PAGE electrophoresis in $12 \%$ polyacrylamide gels (Schagger and von Jagow 1987) using prestained molecular weight standards (New England Biolabs).

\section{Activity assays}

The biochemical characterisation of the purified enzymes was assayed as previously described by Sussman and Avron (1981). The reaction mixture contained $20 \mathrm{mM}$ Tris $-\mathrm{HCl}$ $\mathrm{pH}$ 7.0, $5 \mathrm{mM} \mathrm{MgCl}_{2}$, and $10 \mathrm{mM}$ D-ribose-5-phosphate, 2-deoxy-D-ribose-5-phosphate, 2-deoxy-D-glucose-6-phosphate, D-mannose-6-phosphate, D-fructose-1-phosphate, D-glucose-6-phosphate, DL-glycerol-3-phosphate, D-fructose-6-phosphate, $\alpha$-D-glucose-1-phosphate, $\alpha$-D-mannose1-phosphate, D-fructose-1,6-bisphosphate, $\alpha$-D-glucose-1, 6-bisphosphate or D-erythrose-4-phosphate (R7750, D3126, D8875, M6876, F1127, G7250, G2138, F1502, G9380, M1755, F4757, G5750, E0377, respectively) (Sigma) in a total volume of $1 \mathrm{ml}$. Approximately $25 \mu \mathrm{g} / \mathrm{ml}$ of purified protein was used in the enzymatic reactions. After 15, 30 and $60 \mathrm{~min}$ of incubation with the enzyme, samples of $0.3 \mathrm{ml}$ were withdrawn and added to $0.7 \mathrm{ml}$ phosphate determination mixture. The released inorganic phosphate was determined according to Ames (1966) and the reaction rate was calculated in relation to the amount of enzyme and time. Different substrate concentrations $(0.5-32 \mathrm{mM})$ were used to receive the Michaelis-Menten kinetic parameters $K_{\mathrm{m}}$ and $V_{\mathrm{max}}$. The $k_{\text {cat }}$ was calculated from the equation: $k_{\text {cat }}=V_{\text {max }} /[E]$ ([E] enzyme concentration in molar). To demonstrate the influence of the $\mathrm{pH}$, reaction mixtures with the $\mathrm{pH}$ range of 2-10 were used; the experiments were done at least twice with values differing not significantly.

\section{Northern blots and hybridization}

Northern analyses were performed using approximately $20 \mathrm{mg}$ of total RNA per track. Isolated DNA fragments were nick-translated in the presence of $\alpha-\left[{ }^{32} \mathrm{P}\right] \mathrm{dCTP}$ to be used as probes (Maniatis et al. 1982). Probe AtSgpp cDNA was a 735-bp EcoRI/SalI fragment, which contains the complete AtSgpp coding region. Hybridization was performed in $3 \times$ SSC (saline sodium citrate), $0.05 \%$ PVP, 0.05 Ficoll, $1 \%$ SDS and $50 \mu \mathrm{g} \mathrm{ml}^{-1}$ ssDNA (salmon sperm DNA) (Sigma) at $55^{\circ} \mathrm{C}$. Filters were washed at high stringency $(0.1 \times \mathrm{SSC}$ and $0.5 \% \mathrm{SDS})$ at $55^{\circ} \mathrm{C}$. The experiments were performed more than once and the data shown are representative.

\section{Results}

Isolation of A. thaliana AtGpp-homologous genes

In previous work, the complete sequence of the Arabidopsis genome (The Arabidopsis Genome Initiative 2000) was used for the identification of the two uncharacterised A. thalianaDL-glycerol-3-phosphatase genes. With the known budding yeast GPP1 and GPP2 protein sequences and using the BLAST program (Altschul et al. 1990) two putative A. thaliana, named AtGppl and AtGpp2, were identified, cloned and characterized (Caparrós-Martín et al. 2007). Using a comparative genome approach, four additional homologous genes were detected, with similar scores and general function predicted as phosphatase/phosphohexomutase (unknown At2g38740, putative At4g39970, catalytic/hydrolase At3g48420 and catalytic/hydrolase/ phosphoglycolate phosphatase At2g33255). The virtual isolated genes were cloned by RT-PCR and sequenced.

Gene thin structure

In agreement with the NCBI reported annotations, loci At2g38740, At4g39970, At3g48420 and At2g33255 are encoded on A. thaliana chromosome 2, 4, 3 and 2 as 1,786, 1,751, 1,808 and 1,563-bp length genes with 3, 9, 7 and 4 introns, respectively, with unspliced $5^{\prime}$ and $3^{\prime}$-untranslated regions. The At2g38740, At4g39970, At3g48420 and At2g33255 proteins have 244, 316, 319 and 245 amino acids, a deduced $\mathrm{Mw}$ of $26.7,34.7,34.3$ and $27.5 \mathrm{kDa}$ and pI of 4.6, 5.2, 8.2 and 6.1, respectively. At2g38740 does not have any predicted signal to mitochondrial or chloroplast targeting, while for At4g39970, At3g48420 and At2g33255 its location is expected to be in the chloroplast.

Sequence comparisons

The four plant proteins show significant similarity (28-39\%, sequence identity of $18-23 \%$ ) to A. thalianaDLglycerol-3-phosphatases AtGpp1 and AtGpp2 (Fig. 1). This homology is ubiquitously distributed although, as it would be expected among HAD members, the greatest similarity occurs at the four loops forming the catalytic scaffold of the active site platform framed by the core domain (Zhang et al. 2004; Lu et al. 2005), particularly in the highly conserved sequence motifs by which family members are recognized, contained in loops 1-4 (1, FDxDG; 2, xT/Sx; 3, KPxP; 4, ED, or GDxxxDD), that positions substrate-cofactor binding and catalytic residues that are involved in the core chemistry (Burroughs et al. 2006). Fewer sequence homologies are shared at the predicted cap domain substrate recognition loop 5, responsible 
Fig. 1 AtGpp alignment with its homologous proteins. The amino acid sequence of the A. thaliana DL-glycerol-3phosphatases AtGpp1 and AtGpp2 was compared with their homologous

representatives from $A$. thaliana At2g38740, At4g39970,

At3g48420 and At2g33255. Residues that are identical or similar to AtGpp1 and AtGpp2 are highlighted with asterisks or dots, respectively, and the shared motifs are depicted in bold
AtGpp1
AtGpp2
At 4g39970
At 3948420
At2g38740
At 2933255
AtGpp1
AtGpp 2
At 4939970
At 3948420
At2g38740
At 2933255
AtGpp1
AtGpp2
At 4939970
At 3948420
At 2938740
At 2933255
AtGpp1
AtGpp2
At 4939970
At 3948420
At 2938740
At 2933255

Motif 1

AVNATVTVTDAGRGS ITHVIFDMDGLLLDTE-KFYTEVOEKILARYNKTFD-------W 106 VT-------AGRGS ITHVI FDMDGLLLDTE-KFYTEVQEI ILARFNKKFD--------W 49 -SRVSSPVSALPLRSLEALIFDCDGVILESE-NLHRQAYNDAFSHFDVRCPPSSSESLDW 106 SVGVTCSASSSLTTLPSALLFDCDGVLVDTEKDGHRISFNDTFKERDLNVT--------W 112 --------QLAP LEA I LFDVDGTLCDSD-PIHLIAFQELLQEIGFNNG--------V 57 $---------A K T R L R G V V F D M D G T L T V P V I D F A A M Y R A V L G E D A Y K R I K--------68$

Motif 5

SLKAK-----MMGRKAIEAARLFVD--------ESGISDSLSAEDFIVERESMLQDLFP 152 SLKAK-----MMGRKAIEAARIFVE---------ESGISDSLSAEDFLVERESMLQDLFP 95 SLEFYDKFQNLVGGGKPKMRWYFKENGWPTSTIFDSPPQNDDDRAKLIDTLQDWKTERYK 166 DVDLYGELL-KIGGGKERMTAYFNKVGWP-----EKAPKDEAERKEFIAGLHKQKTELFM 166 PIDEKFFVENIAGKHNSEIALLLFP-----------DDVSRGLKFCDEKEALYRKIVA 104 $--------A E S P S G I D I L H H I E S--------W S P D K Q Q K A Y E I I A D Y E K Q G I D K L Q 108$

Motif 2

-----TSDLMPGASRLLRHLHGKGIPICIATGTHTRHFDLKTORHRELFS LMHHVVRGD 206 -----TSELMPGASRLIKHLHVKNIPICIATGTHTRHYDLKTQRHRELFSLMHHVVRGD 149 EIIKSGSVEPRPGVIRLMDEAKAAGKKLAVCSAATKSSVILCLENLIDIERFOGLDCFLA 226 VLIEKKLLPLRPGVAKLVDQALTNGVKVAVCSTSNEKAVSAIVSCLLGPERAEKIKIFAG 226 -----EKIKPLDGLIKLTKWIEDRGLKRAAVTNAPKENAELMIS-KLGLTDFFQAVILG- 157 --------IMPGTAELCGFLDSKKIKRGLITRNVQKAIDIFHQRFEVIFSPALG----- 154 Motif 3

Motif 4

DPEVKEGKPAPDGFLAASRRFEDGPVDPRKVLVFEDAPSG-VQAAKNAG-MNVIMVPDSR 264 DPEVKQGKPAPDGFLAAARRFKDGPVDSQKVLVFEDAPSG-VLAAKNAG-MNVVMVPDPR 207 GDDVKEKKPDPSIYITAAEKLG---VSVKDCLVVEDSVIG-LQAATKAG-MSCVITYTSS 281 -DVVPKKKPDPAIYNLAAETLG---VDPSKCVVVEDSAIG-LAAAKAAG-MTCIVTKSGY 280 -SECEFPKPHPGPYLKALEVLN---VSKEHTLVEEDSISG-IKAGVAAG-MPVIGLTTGN 211 -REFRPYKPNPDPLLHICSTWD---IQPNEVMMVGDSLKDDIACGKRAGAFTCLLDETGR 210

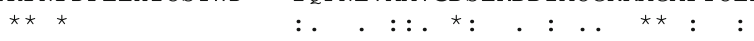

for the chemical diversification within the family, apart from the stringently conserved loop marker Gly (G), flanked by Lys/Arg (K/R) and residues, whose side chains contribute to the catalytic site, probably operating in domain-domain binding, active-site desolvation and/or catalysis (Lahiri et al. 2004), that must provide the signature-based substrate specificity.

Protein expression, purification and substrate screening

To examine the phosphatase activity, the AtGpp homologous At2g38740, At4g39970, At3g48420 and At2g33255 (26.7, 34.7, 34.3 and $27.5 \mathrm{kDa}$, respectively) were expressed as MBP (42.7 kDa) fusion proteins, purified from the corresponding $E$. coli clones, by amylose affinity chromatography, and their phosphatase activity determined in the presence of $\mathrm{Mg}^{2+}$ ions (Fig. 2).

Presuming a connected sequence-based assignment of function on targeting substrates with similar structural characteristics, the four purified proteins (Fig. 2a) were investigated for DL-glycerol-3-phosphatase activity and against a set of related phosphoesters such as D-fructose-6phosphate, D-fructose-1-phosphate, D-fructose-1,6-bisphosphate, D-glucose-6-phosphate, $\alpha$-D-glucose-1-phosphate, 2-deoxy-D-glucose-6-phosphate, D-mannose-6-phosphate, and $\alpha$-D-mannose-1-phosphate. Pi release, from hydrolyzed substrates, was found in assays with MBP-At2g38740, whereas there was no hydrolytic activity detectable for MBP-At4g39970, MBP-At3g48420, or MBP-At2g33255
(Fig. 2b) even though their predicted cap domains are remarkably similar in fold (result not shown). Yet it cannot be discounted that the examined inactive proteins were expressed and purified in a functionally folded form. Table 1 represents the in vitro activity of purified At $2 \mathrm{~g} 38740$ on various organic phosphoesters. The plant enzyme, hereafter called A. thaliana phosphosugar phosphatase AtSgpp, shows a broad-range sugar phosphate phosphatase affinity. The activity is preferentially detectable on D-ribose-5-phosphate, 2-deoxy-D-ribose-5-phosphate, 2-deoxy-D-glucose-6-phosphate, and D-mannose-6-phosphate; lower activity is observed on D-fructose-1-phosphate, D-glucose-6-phosphate, DL-glycerol-3-phosphate, and D-fructose-6-phosphate. The phosphorylated compounds $\alpha$-D-glucose-1-phosphate, $\alpha$-D-mannose-1-phosphate, D-fructose-1,6-bisphosphate, $\alpha$-D-glucose-1,6-bisphosphate, and D-erythrose-4-phosphate were also tested and showed no significant activity.

Kinetic analysis

AtSgpp protein was further investigated for substrate specificity. The apparent $K_{\mathrm{m}}$ and $V_{\max }$ values of MBPAtSgpp for phosphoesters/ $\mathrm{Mg}^{2+}$ were determined by nonlinear regression from spectrophotometric data (Fig. 3). The corresponding slopes of the Lineweaver-Burk plot fall into two differentiated categories, lower $K_{\mathrm{m}} / V_{\max }$ values are obtained for D-ribose-5-phosphate, 2-deoxyD-ribose-5-phosphate, 2-deoxy-D-glucose-6-phosphate, and D-mannose-6-phosphate (Fig. 3a), than for those of 


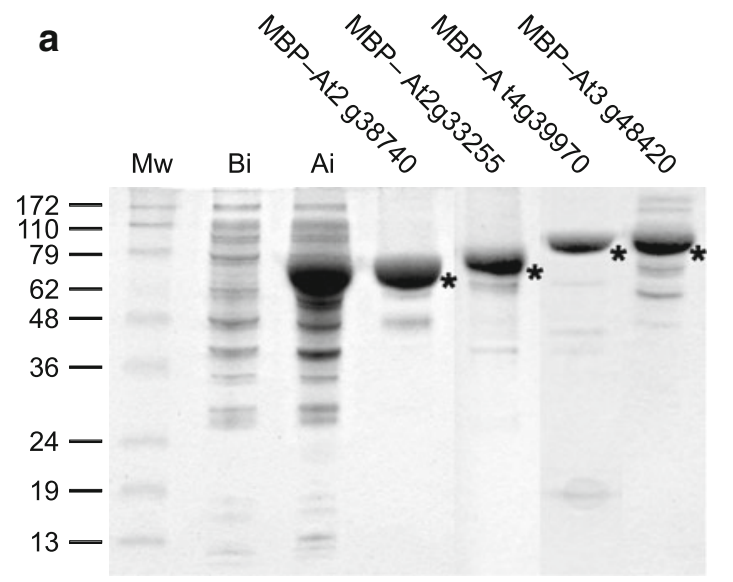

$\begin{array}{lcccc}\text { b } & \text { At2g38740 } & \text { At2g33255 } & \text { At4g39970 } & \text { At3g48420 } \\ \text { G3P } & + & - & - & - \\ \mathrm{F} 6 \mathrm{P} & + & - & - & - \\ \mathrm{F} 1 \mathrm{P} & + & - & - & - \\ \mathrm{F} 1,6 \mathrm{P} & - & - & - & - \\ \mathrm{G} 6 \mathrm{P} & + & - & - & - \\ \mathrm{G} 1 \mathrm{P} & - & - & - & - \\ \text { 2DG6P + } & - & - & - \\ \mathrm{M} 6 \mathrm{P} & + & - & - & - \\ \mathrm{M} 1 \mathrm{P} & - & - & - & -\end{array}$

Fig. 2 Purification and hydrolytic activity of AtGpp homologues. a, b SDS-PAGE of purified MBP-fusion proteins by amylose affinity chromatography. Prestained molecular weight marker in $\mathrm{kDa}(M w)$; MBP-At2g38740 before induction (Bi); MBP-At2g38740 after induction $(A i)$; partially purified MBP-proteins: At2g38740, At2g33255, At4g39970, and At3g48420 (a). Hydrolytic activity of the AtGpp homologous At2g38740, At4g39970, At3g48420, and At2g33255 on DL-glycerol-3-phosphate $(G 3 P)$, D-fructose-6-phosphate $(F 6 P)$, D-fructose-1-phosphate $(F 1 P)$, D-fructose-1,6-bisphosphate $(F 1,6 P)$, D-glucose-6-phosphate $(G 6 P), \alpha$-D-glucose-1-phosphate $(G 1 P)$, 2-deoxy-D-glucose-6-phosphate (2DG6P), D-mannose-6-phosphate $(M 6 P)$, and $\alpha$-D-mannose-1-phosphate $(M 1 P)$ (b). Purified MBP fusion proteins are indicated by asterisks. (+) Positive and (-) negative hydrolysis

D-fructose-1-phosphate, D-glucose-6-phosphate, DL-glycerol-3-phosphate, and D-fructose-6-phosphate (Fig. 3b).

The catalytic hydrolysis occurs with inconspicuous specificity and efficiency; the values of the substrate specificity constant $k_{\mathrm{cat}} / K_{\mathrm{m}}$ for D-ribose-5-phosphate, 2-deoxy-D-ribose5-phosphate, 2-deoxy-D-glucose-6-phosphate, D-mannose-6phosphate, D-fructose-1-phosphate, D-glucose-6-phosphate, DL-glycerol-3-phosphate, and D-fructose-6-phosphate are in the range of 2.5-10.7 $\times 10^{3} \mathrm{M}^{-1} \mathrm{~s}^{-1}$, being the highest $k_{\text {cat }} /$ $K_{\mathrm{m}}$ value for D-ribose-5-phosphate $\left(10.7 \times 10^{3} \mathrm{M}^{-1} \mathrm{~s}^{-1}\right)$ and the lowest for D-fructose-6-phosphate $\left(2.5 \times 10^{3}\right.$ $\mathrm{M}^{-1} \mathrm{~s}^{-1}$ ) (Table 2).
Table 1 Phosphatase activity of purified AtSgpp on various organic phosphoesters

\begin{tabular}{lc}
\hline Substrate & Relative activity \\
\hline Ribose-5-phosphate & 100 \\
2-Deoxyribose-5-phosphate & 93 \\
2-Deoxyglucose-6-phosphate & 91 \\
Mannose-6-phosphate & 85 \\
Fructose-1-phosphate & 77 \\
Glucose-6-phosphate & 70 \\
DL-Glycerol-3-phosphate & 55 \\
Fructose-6-phosphate & 51 \\
Glucose-1-phosphate & - \\
Mannose-1-phosphate & - \\
Fructose-1,6-bisphosphate & - \\
Glucose-1,6-bisphosphate & - \\
Erythrose-4-phosphate & -
\end{tabular}

The reaction mixture contained: $20 \mathrm{mM}$ Tris-HCI ( $\mathrm{pH} 7.0), 5 \mathrm{mM}$ $\mathrm{MgCI}_{2}, 10 \mathrm{mM}$ substrate (different phosphoesters) and $25 \mu \mathrm{g} / \mathrm{ml}$ of the purified protein. Reaction temperature: $32^{\circ} \mathrm{C}$. The enzyme activity with D-ribose-5-phosphate was set to 100
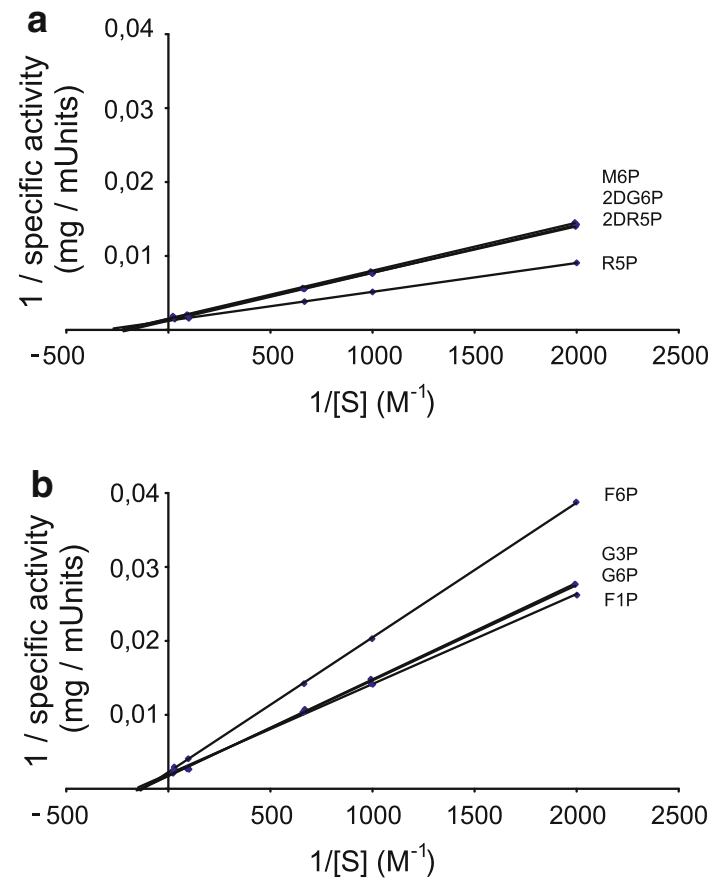

Fig. 3 AtSgpp phosphatase activity profile. a, b The apparent $K_{\mathrm{m}}$ and $V_{\max }$ of the AtSgpp phosphatase for D-ribose-5-phosphate (R5P), 2-deoxy-D-ribose-5-phosphate (2DR5P), 2-deoxy-D-glucose-6-phosphate (2DG6P), D-mannose-6-phosphate (M6P) (a); for D-fructose-1phosphate (F1P), D-glucose-6-phosphate (G6P), DL-glycerol-3-phosphate (G3P), D-fructose-6-phosphate (F6P) (b). The reaction mixture contained: $20 \mathrm{mM}$ Tris- $\mathrm{HCl}(\mathrm{pH} 7.0), 5 \mathrm{mM} \mathrm{MgCl} 2$, the indicated concentration of substrate, $25 \mu \mathrm{g} / \mathrm{ml}$ of AtSgpp. Reaction temperature: $32{ }^{\circ} \mathrm{C}$ 
Table 2 Kinetics parameters of the phosphatase activity of purified AtSgpp on various organic phosphomonoesters

\begin{tabular}{lllll}
\hline Substrate & $V_{\max }\left(\mu \mathrm{mol} \mathrm{s}{ }^{-1}\right)$ & $K_{\mathrm{m}}(\mathrm{M})$ & $k_{\mathrm{cat}}\left(\mathrm{s}^{-1}\right)$ & $k_{\mathrm{cat}} / K_{\mathrm{m}}\left(\mathrm{M}^{-1} \mathrm{~s}^{-1}\right)$ \\
\hline D-Ribose-5-phosphate & $(13.8 \pm 0.5) \times 10^{-3}$ & $(3.6 \pm 0.1) \times 10^{-3}$ & $(3.9 \pm 0.3) \times 10$ & $10.7 \times 10^{3}$ \\
2-Deoxy-D-ribose-5-phosphate & $(11.9 \pm 0.3) \times 10^{-3}$ & $(4.5 \pm 0.3) \times 10^{-3}$ & $(3.3 \pm 0.2) \times 10$ & $7.3 \times 10^{3}$ \\
2-Deoxy-D-glucose-6-phosphate & $(11.8 \pm 0.6) \times 10^{-3}$ & $(4.6 \pm 0.5) \times 10^{-3}$ & $(3.3 \pm 0.1) \times 10$ & $7.2 \times 10^{3}$ \\
D-Mannose-6-phosphate & $(11.6 \pm 0.4) \times 10^{-3}$ & $(4.9 \pm 0.6) \times 10^{-3}$ & $(3.2 \pm 0.6) \times 10$ & $6.7 \times 10^{3}$ \\
D-Fructose-1-phosphate & $(9.2 \pm 0.3) \times 10^{-3}$ & $(6.9 \pm 0.1) \times 10^{-3}$ & $(2.6 \pm 0.4) \times 10$ & $3.7 \times 10^{3}$ \\
D-Glucose-6-phosphate & $(8.7 \pm 0.2) \times 10^{-3}$ & $(7.1 \pm 0.3) \times 10^{-3}$ & $(2.4 \pm 0.5) \times 10$ & $3.4 \times 10^{3}$ \\
DL-Glycerol-3-phosphate & $(8.3 \pm 0.2) \times 10^{-3}$ & $(7.3 \pm 0.2) \times 10^{-3}$ & $(2.3 \pm 0.3) \times 10$ & $3.1 \times 10^{3}$ \\
D-Fructose-6-phosphate & $(6.9 \pm 0.1) \times 10^{-3}$ & $(7.7 \pm 0.4) \times 10^{-3}$ & $(1.9 \pm 0.2) \times 10$ & $2.5 \times 10^{3}$ \\
\hline
\end{tabular}

The reaction mixture contained: $20 \mathrm{mM}$ Tris-HCI (pH 7.0), $5 \mathrm{mM} \mathrm{MgCI}_{2}, 10 \mathrm{mM}$ substrate (different phosphomonoesters) and $25 \mu \mathrm{g} / \mathrm{ml}$ of the purified protein. Reaction temperature: $32{ }^{\circ} \mathrm{C}$
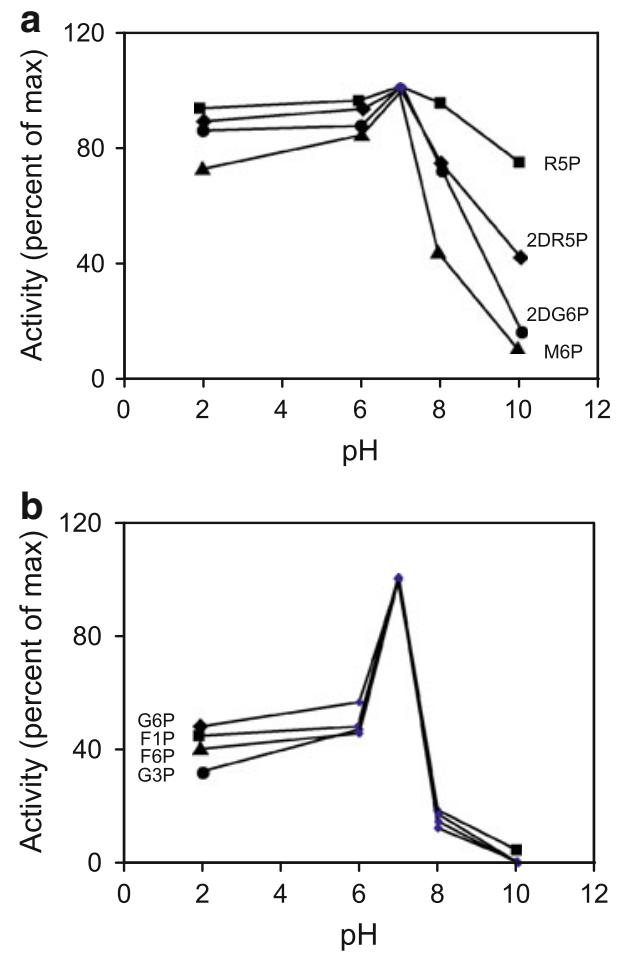

Fig. 4 AtSgpp $\mathrm{pH}$ dependence. a, b Influence of $\mathrm{pH}$ on the phosphatase activity of AtSgpp for D-ribose-5-phosphate (R5P, solid square), 2-deoxy-D-ribose-5-phosphate (2DR5P, solid diamond), 2-deoxy-D-glucose-6-phosphate (2DG6P, solid circle), D-mannose-6phosphate (M6P, solid triangle) (a); D-fructose-1-phosphate (F1P, solid square), D-glucose-6-phosphate (G6P, solid diamond), DL-glycerol-3-phosphate (G3P, solid circle), D-fructose-6-phosphate (F6P, solid triangle) (b). The reaction mixture contained: $20 \mathrm{mM}$ Tris- $\mathrm{HCl}$ (the indicated $\mathrm{pH}$ ), $5 \mathrm{mM} \mathrm{MgCl}_{2}, 10 \mathrm{mM}$ substrate, 25 $\mu \mathrm{g} / \mathrm{ml}$ of AtSgpp. Reaction temperature: $32{ }^{\circ} \mathrm{C}$

$\mathrm{pH}$ rate profile determinations

Like most HAD phosphatases, AtSgpp developed optimal activity toward natural substrates at neutral $\mathrm{pH} 7.0$ (Fig. 4). Interestingly, the pH dependence of MBP-AtSgpp catalysis on D-ribose-5-phosphate, 2-deoxy-D-ribose-5phosphate, 2-deoxy-D-glucose-6-phosphate, and D-mannose- 6-phosphate (Fig. 4a), again differs from those of D-fructose1-phosphate, D-glucose-6-phosphate, DL-glycerol-3-phosphate, and D-fructose-6-phosphate (Fig. 4b). It is striking that the MBP-AtSgpp targeting per D-ribose-5-phosphate does not significantly change over the entire $\mathrm{pH}$ range, whilst it decreases as $\mathrm{pH}$ increases in the case of 2-deoxy-D-ribose-5phosphate, 2-deoxy-D-glucose-6-phosphate, and D-mannose6-phosphate. However, MBP-AtSgpp affinity abruptly drops, following a graph bell-shaped, on D-fructose-1-phosphate, D-glucose-6-phosphate, DL-glycerol-3-phosphate, and D-fructose-6-phosphate, when reaching an acidic or basic $\mathrm{pH}$. This contrasting behavior may result from $\mathrm{pH}$-dependent differences, in active site desolvation and conformational entropy, upon substrate binding and the induced cap closure.

\section{AtSgpp gene expression}

To gain insight into the physiological importance of the AtSgpp hydrolytic activity in plants, the expression of the A. thalianaAtSgpp gene was analyzed, by Northern hybridization, in different tissues and under stress conditions (Fig. 5). In all blot experiments a band was detected corresponding in size to the AtSgpp transcripts. The AtSgpp pattern of expression was examined in roots, shoots, leaves, flowers and developing siliqua (Fig. 5a). Transcripts of the gene were detectable on northern blots from all of these organs. High expression was found in flowers, pointing to a prominent role of this gene during floral development. AtSgpp mRNA accumulation was also observed in shoots, siliqua, roots and the lowest in leaves. AtSgpp expression was also examined in seedling subjected to salt $(100 \mathrm{mM}$ $\mathrm{NaCl}$ and $15 \mathrm{mM} \mathrm{LiCl}$ ), osmotic (200 $\mathrm{mM}$ sorbitol) or oxidative ( $5 \mathrm{mM} \mathrm{H}_{2} \mathrm{O}_{2}$ and $1 \mu \mathrm{M}$ methyl viologen) stresses (Fig. 5b). AtSgpp expression seems to be affected in seedlings that undergo all these abiotic stresses.

More detailed information about the AtSgpp pattern of expression was obtained from the microarrays data bank Genevestigator (Zimmermann et al. 2004). Expression during development is medium, declining to low with 

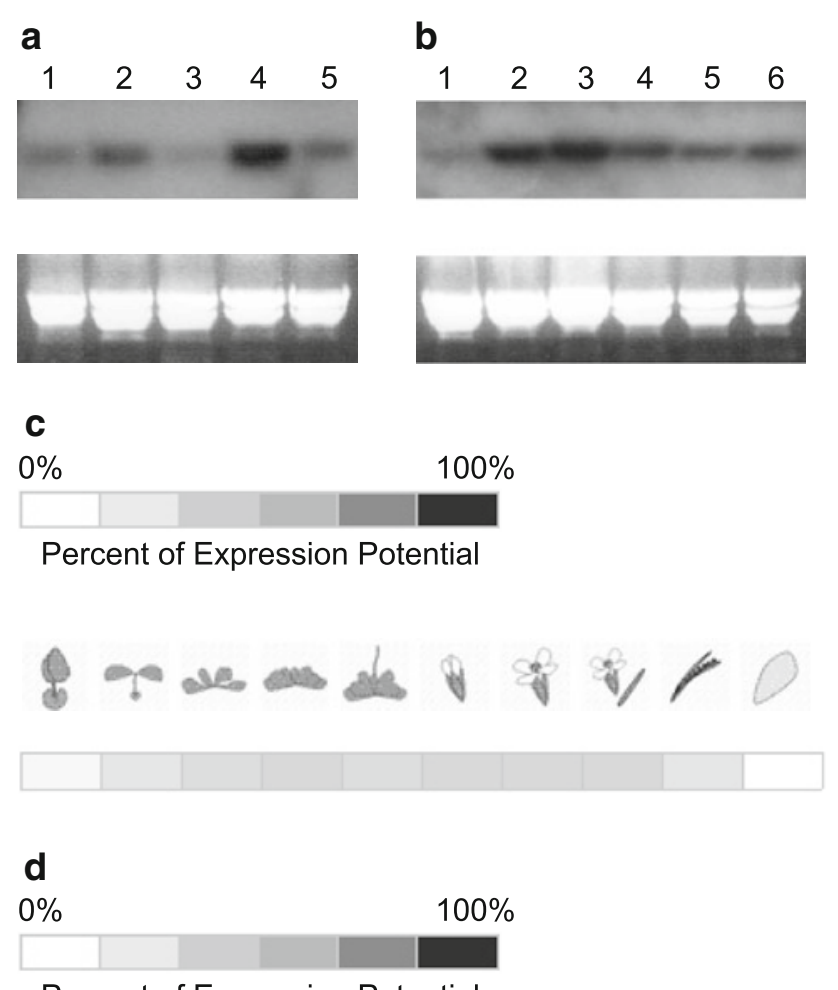

Percent of Expression Potential

Sepal

Guard cell protoplast

Cell culture / primary cell

Root cortex protoplast

Petal

Cauline leaf

Stamen

Senescent leaf

Root epidermal atrichoblast protoplast

Replum

Lateral root

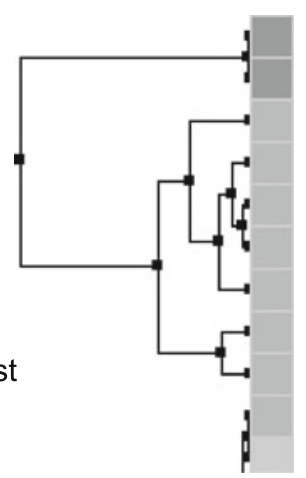

Fig. 5 Expression of AtSgpp mRNA during development and under stress treatment. a, b Northern blot of Arabidopsis RNA probed with radiolabelled AtSgpp cDNA. c, d AtSgpp pattern of expression from the microarrays data bank Genevestigator. a From different tissues: 1 root, 2 shoot, 3 leaves, 4 flowers, 5 siliqua. b From 12-day-old seedling cultivated on MSS medium: 1 control, $2 \mathrm{MSS}+100 \mathrm{mM}$ $\mathrm{NaCl}, 3 \mathrm{MSS}+200 \mathrm{mM}$ sorbitol, $4 \mathrm{MSS}+15 \mathrm{mM} \mathrm{LiCl}, 5$ MSS $+5 \mathrm{mM} \mathrm{H} \mathrm{H}_{2} \mathrm{O}_{2}$ and $6 \mathrm{MSS}+1 \mu \mathrm{M}$ methyl viologen. In Northern blots, ethidium bromide-stained RNA was used as loading control. c Expression profile over time throughout the live cycle of the Arabidopsis. d Partial hierarchical clustering displaying the level of the anatomical expression across of tissue types

senescence (Fig. 5c), while the anatomical expression is in most plant organs, leading to their hierarchical clustering in the sepals and the guard cell protoplasts (Fig. 5d). The quantitative transcriptomic analysis also shows that expression of locus At $2 g 38740$ is perturbed by abiotic and biotic stresses, being up-regulated by cold, heat, oxidative, osmotic, salt, drought, hypoxia, genotoxic, and bounding, as well as in response to pathogens (not shown).

Signature sequence substrate specificity loop 5

While the catalytic core is superimposable, the cap domain has evolved through the evolution, remaining responsible of targeting diversification among members of the subfamily. The cap contains the so-called substrate specificity loop 5 that provides the signature sequence motif 5 which, in the closed conformation of the enzyme, defines the active site and its specific chemistry. Core sequence motifs 1 and 2 determine the boundaries of the cap sequence segment in subfamily I, and the conserved Gly identifies the loop 5 motif within it, allowing detection of cap domain-derived active site residues, in the absence of a three-dimensional structure (Lahiri et al. 2004). Using this effective tool for function assignment together with their predicted structure, the substrate specificity loop 5 from known HAD phosphohydrolases GPP (Norbeck et al. 1996), DOG (Rández-Gil et al. 1995), AtGpp (CaparrósMartín et al. 2007), AtSgpp and unknown homologous representatives, were compared (Fig. 6).

In baker's yeast subclass I DL-glycerol-3-phosphatases GPP1 and GPP2 and 2-deoxy-D-glucose-6-phosphatases DOG1 and DOG2, their putative shared motif 5 (SHGW/ AR) presents a single shift of Trp (W56 in GPP1 and GPP2) per Ala (A49 in DOG1 and DOG2) which could impart substrate specificity, while between yeast GPP1 and GPP2 and its plant counterparts AtGpp1 and AtGpp2, just the central Gly (G55 in GPP1 and GPP2 and G57 in AtGpp2), essential in determining the loop 5 conformation and flexibility (Lahiri et al. 2004), and surrounding Lys (R57 in GPP1 and GPP2 and R58 in AtGpp2) are conserved (Fig. 6a). By contrast, the motif 5 is well preserved between genuses within the kingdom itself (Fig. 6b, c). Lack of shared homology is also appreciated among phosphosugar phosphatases, subfamily IIB bacterial BT4131 and subclass I plant AtSgpp, despite their analogous catalysis and substrate specificity (not shown).

\section{Discussion}

The objective of this study was to search for the sequencefunction relationship, which drives the substrate discrimination between HAD phosphohydrolases subfamily members. Application of general enzymatic screenings and substrate profiling is a useful tool to discover new enzymes (Kuznetsova et al. 2005); likewise, the substrate prediction of unknown HAD hydrolases and their confirmed function, by applying the predicted substrates to the purified protein, has been an elegant strategy that has been successfully 
a

DOG1

DOG2

GPP1

GPP2

AtGpp1

AtGpp2

b

AtGpp2

Vitis

Oryza

Sorghum

Zea

Chlamydomonas

Homo

C

Zea

Sorghum

Oryza

At Sgpp

Vitis

Pseudomonas
Chlamydomonas

Motif 5

----MAEFSADLCLFDLDGTIVSTTVAAEKAWTKLCYEYG-VDPSELFKHSHGARTQEV 54 -MPOFSVDLCLFDLDGTIVSTTTAAESAWKKLCRQHG-VDPVELFKHSHGARSOEM 54 PLTTKPLSLKINAALFDVDGTIIISQPAIAAFWRDFGKDKPYFDAEHVIHISHGWRTYDA 61 GLTTKPLSLKVNAALFDVDGTIIISQPAIAAFWRDFGKDKPYFDAEHVIQVSHGWRTFDA 61 VTVTDAGRGS ITHVIFDMDGLLLDTEKFYTEVQEKILARYNKTFDWSLKAKMMGRKAIEA 120 ----AGRGS ITHVIFDMDGLLLDTEKFYTEVQEI I LARFNKKFDWSLKAKMMGRKAIEA 63 $: \star \star * \star \star::$

Motif 5

FDMDGLLLDTEKFYTEVQEI ILARFNKKFDWSLKAKMMGRKAIEAARIFVEESGIS-DSL 77 FDMDGLLLDTEKFYTEVQEI ILARYNKTFDWSLKAKMMGKKAIEAARVFVEETGIS-DSL 70 FDMDGLLLDTEGFYTEVQEKILARYGKVFDWSLKAKMMGKKATESARIFVDECGLD-GLL 80 FDMDGLLLDTEGFYTEVQEKILARYGKVFDWSLKAKMMGKKATESARIFVDECGLN-GLL 113 FDMDGLLLDTEGFYTEVQEKILARYDKVFDWSLKAKMMGKKAAESARIFVDECGLN-GLL 117 --MDGLLLDTEGAYTVAQORILDRFGRKFTWELKAKMMGROALDAARVLCEDLKLTPEEI 58 --MDGLLLDTERLYSVVFQE ICNRYDKKYSWDVKS LVMGKKALEAAQI I IDVLQLP---M 55

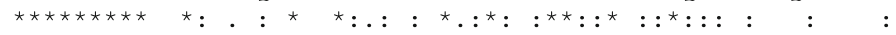

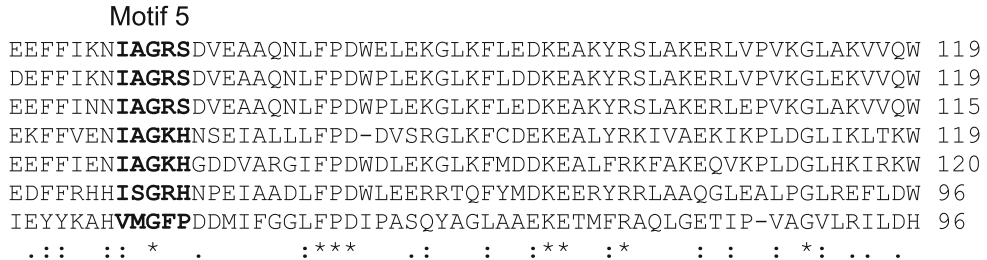

Fig. 6 Comparison of the signature sequence motif 5. a The amino acid sequence of $A$. thalianaDL-glycerol-3-phosphatases AtGpp1 and AtGpp2 was compared with homologous representatives from S. cerevisiaeDL-glycerol-3-phosphatases GPP1 and GPP2 and 2-deoxy-D-glucose-6-phosphatases DOG1 and DOG2. b, cArabidopsisDL-glycerol-3-phosphatase AtGpp2 and phosphosugar phosphatase AtSgpp were also compared with orthologous candidates from other genus by NCBI BLink: Vitis (CBI17840), Oryza (BAD05444), Sorghum (EES14772), Zea (ACF85466), Chlamydomonas

(EDP00037), and Homo (AAH12494) (b) and Vitis (CBI33932), Oryza (BAD36300), Sorghum (EER89058), Zea (ACF83536), Chlamydomonas (EDP04776), and Pseudomonas (EGH95925) (c), respectively. Conserved residues are labeled with asterisks or dots and the putative shared motifs in bold. In the absence of a three-dimensional structure, cap domain-derived active site residues were identified by the conjoined information from predicted 3D models, the boundaries of the cap sequence segment defined by motifs 1 and 2, and the conserved Gly $(G)$ that identify the loop 5 motif within this segment

carried out (Lu et al. 2005). Following sequence-function analysis, as those used in the characterization of ArabidopsisDL-glycerol-3-phosphatases AtGpp1 and AtGpp2 (Caparrós-Martín et al. 2007), the physiological substrate specificity of the closest AtGpp homologues At2g38740, At4g39970, At3g48420, and At2g33255 was analyzed. Rather than for their genomic context, loci were chosen for the remarkable shared similarity of the encoding genes. In the spirit of the initial strategy it was to contribute to the hypothesis that might be possible to infer guidelines for function assignment within the HAD family, based on the sequence data alone (Lahiri et al. 2004); abounding into the syllogism that, if the cap domain defines substrate specificity and the physiological substrate determines the biochemical function, logically the relationship between cap domain and substrate structure is the essential clue to the sequence-based assignment of its function (Tremblay et al. 2006).

The AtSgpp catalytic hydrolysis of cyclic sugars occurs with inconspicuous specificity and efficiency, $k_{\mathrm{cat}} / K_{\mathrm{m}}$ in the range of $2.5-10.7 \times 10^{3} \mathrm{M}^{-1} \mathrm{~s}^{-1}$. Such a modest catalysis and substrate promiscuity has been reported by the hexose phosphate phosphatase HPP (BT4131 from Bacteroides thetaiotaomicron VPI-5482), whose catalytic efficiency is low: $k_{\mathrm{cat}} / K_{\mathrm{m}} \sim 1 \times 10^{3} \mathrm{M}^{-1} \mathrm{~s}^{-1}$ compared to the $1 \times 10^{6}$ to $1 \times 10^{8} \mathrm{M}^{-1} \mathrm{~s}^{-1}$ "gold-standard" for single-substrate enzymes involved in primary metabolism (Lu et al. 2005). Bacterial BT4131 is a member of the haloalkanoate dehalogenase superfamily (subfamily IIB) with a C2B-type cap domain, whilst the expected structure of the plant enzyme yet enrolls AtSgpp as a member of the subfamily I with the C1-type cap domain. Besides the lax specificity for the ring size and stereochemistry of the sugar phosphate, AtSgpp shares with BT4131 the ability to discriminate between isomers that do not accommodate into the active site solvent cage, such as D-glucose-1-phosphate or $\alpha$-D-mannose-1-phosphate and, to a lesser extent, D-fructose-6-phosphate. As suggested for other HAD monophosphatases (Kuznetsova et al. 2006; Tremblay et al. 2006), the kinetic parameters of AtSgpp demonstrated an overlapping catabolic activity, with undefined boundaries on physiologically related substrates that, in this case, point to a role in the metabolic regulation of phosphosugars intermediates of the glycolysis and the pentose phosphate pathway. The activity of AtSgpp on 2-deoxy-D-glucose-6phosphate also arouses interest. 2-deoxy-D-glucose is a 
non-metabolizable analog of glucose that becomes toxic after its phosphorylation to 2-deoxy-D-glucose-6-phosphate. So far, no plant analogous to the yeast DOG1 and DOG2 genes (Rández-Gil et al. 1995), which dephosphorylate 2-deoxyglucose-6-phosphate, has been found. However, DOG2 transgenic over-expression in tobacco improves the tolerance to the deleterious effects of 2-deoxy-D-glucose on growth, chlorophyll content and the expression of genes related to photosynthesis (Cutanda 2003). In vitro phosphatase activity against 2-deoxyglucose-6-phosphate has also been observed in E. coli YniC (HAD1) protein; E. coli YniC-overproducing strain grew well in the presence of 2-deoxy-D-glucose, demonstrating that the 2-deoxy-D-glucose-6-phosphatase activity of YniC (HAD1), plays an important role for the resistance of E. coli cells to 2-deoxyglucose (Kuznetsova et al. 2006).

Substrate binding regulates, by the hinge motion of the solvated domain linkers, the conformational cap closed/ open interconversion. In the cap-closed conformation, cap and core domain interfaced and residues from the cap substrate specificity loop enter the active site, participating in substrate binding and catalysis, whereas the cap-open conformation allows the access to the active site of the solvent, facilitating the release of the product (Morais et al. 2004). Similarly, as previously reported for subclass I phosphonatase catalysis (Zhang et al. 2002; Morais et al. 2004; Lahiri et al. 2006), one could also suggest that the substrate specificity loop of AtSgpp contributes the catalytic Lys (K71) residue to form a Schiff base with the substrate. Proton dissociation from the K71 in the cap domain versus protonation of His (H72) would be required for the AtSgpp cap-core domains closure. Together with the ionization of several enzyme and substrate groups, the loss of activity at low $\mathrm{pH}$ could be due to the protonation of $\mathrm{H} 72$ and at high $\mathrm{pH}$ to deprotonation of K71. Thus, extreme $\mathrm{pH}$ would modify the intramolecular protontransfer, introducing a change in the electron density of the system and on the stability of the phosphoaspartate intermediate compound. This being the case, the $\mathrm{pH}$ dependency of the AtSgpp chemistry on D-ribose-5-phosphate $/ \mathrm{Mg}^{2+}$ would reflect a narrower chemoselectivity in the active site than those conformed by the remaining ligands tested.

Similar pattern of AtSgpp gene expression, in all Arabidopsis organs, have been shown for DL-glycerol-3-phosphatase AtGpp (Caparrós-Martín et al. 2007), with transcripts particularly abundant in developing siliqua. Particularly interesting is the induced expression under nitrogen and iron deficiency and the abscisic acid-induced and reactive oxygen species-dependent expression in guard cell (Böhmer and Schroeder 2011). Foremost, the greatest expression is observed under $\mathrm{Pi}$ starvation and cyclopentenone oxylipins induction. Oxylipins induce the expression of genes related to detoxification, stress responses, and secondary metabolism, when accumulated in response to stress stimuli such as wounding and pathogen infection, what has been attributed to an increase in ROS (Mueller et al. 2008). Coincidentally, a housekeeping function has also been assigned to the hydrolyzing activity of AtCoase, a pyrophosphatase from Arabidopsis thaliana which cleaves coenzyme-A to $4^{\prime}$-phosphopantetheine and $3^{\prime}, 5^{\prime}$-adenosinediphosphate; the CoA cleaving enzyme, whose ubiquitous expression improves plant development, is a member of the Nudix hydrolases, pyrophosphatases that hydrolyze nucleoside diphosphates (Kupke et al. 2009).

Since the referred signature sequence motif 5 drives the substrate targeting, and consequently may be used as protein tagging, from the sequence motif comparisons, it would be worth considering that, within the same species a unique point mutation Trp/Ala (W/A) may be sufficient to discriminate between structural related substrates, pivoting the stereospecificity for DL-glycerol-3-phosphate or 2-deoxy-D-glucose-6-phosphate. In contrast, orthologous enzymes as DL-glycerol-3-phosphatases, from different kingdoms, differ in their patterns. Most intriguing, in the evolutionary divergence within the HAD family, is the ability of analogous Bacteria-BT4131 and Plantae-AtSgpp to perform similar function with dissimilar topology and location of the cap domain. Considering that, although both the I and II subfamilies act predominantly on small encapsulated substrates (Allen and Dunaway-Mariano 2004), in subfamily I the small $\alpha$-helical-bundle C1-type cap is inserted between loops 1 and 2 of the core domain, whereas in subfamily IIB the larger $\beta$-sandwich C2B-type is between loops 2 and 3 (Selengut and Levine 2000; Shin et al. 2003); moreover, BT4131 uses two substrate specificity loops in substrate recognition (Lu et al. 2005).

In conclusion, this paper reports the enzymatic characterization of A. thaliana locus At2g38740. Rescued from the HAD unknown members and hereafter named as phosphosugar phosphatase AtSgpp, its expected structure lists the enzyme as being a member of the HAD subfamily I C1-type cap domain. Extensive substrate screening reveals that AtSgpp presents substrate promiscuity, with broad-range sugar phosphate phosphatase activity, preferentially detectable on D-ribose-5-phosphate and also 2-deoxy-D-ribose-5-phosphate, 2-deoxy-D-glucose-6-phosphate, D-mannose-6-phosphate, D-fructose-1-phosphate, D-glucose-6-phosphate, DL-glycerol-3-phosphate, and D-fructose-6-phosphate. The kinetic parameters show a humble specificity and efficiency that resemble those of the bacterial BT4131, which is a member of the HAD subfamily IIB with a C2B-type cap domain. BT4131 biochemical function was identified by the conjoined information from residues stationed on substrate specificity 
loops, the active site solvent cage and the genome context of the encoding gene ( $\mathrm{Lu}$ et al. 2005) while for AtSgpp, instead of the genomic context, was the close homology with ArabidopsisDL-glycerol-3-phosphatase AtGpp who designed the sugar phosphoesters screening. AtSgpp phosphatase activity is optimal at $\mathrm{pH} 7.0$, albeit fairly $\mathrm{pH}$ independent on D-ribose-5-phosphate cleaving. At present, it is not known if the ribose ester is the main natural hydrolyzing substrate of AtSgpp, or if it could be another non-screened candidate. Also, if using different assay conditions, the Arabidopsis homologous At4g39970, At3g48420 and At2g33255 will cleave sugar phosphomonoesters, or if they hydrolyze different substrates. AtSgpp is ubiquitously expressed throughout development and its expression is affected by abiotic and biotic stresses, being the greatest under Pi starvation and cyclopentenone oxylipins induction. Therefore, taking into account both the activity and the expression data, the AtSgpp physiological function appears to be somehow related to housekeeping detoxification, sugar-phosphate modulation and in maintaining the homeostatic balance of Pi in the cell. Another aim of this work was to provide further information as to the relationship that binds the function to the substrate specificity loop, to contribute to answer the formerly enunciated question about how then do HAD phosphohydrolases distinguish their substrates? It could be claimed that functional peers can be thereby identified by their cap domain signature-sequence motif, though this function assignment would remain constrained to a single kingdom.

Acknowledgments We acknowledge Professors Montserrat Pagès (CSIC Barcelona, Spain), Thomas Kupke (University of Heidelberg, Germany) and Manuel Hernández (University Polytechnic of Valencia, Spain) for their warm support. We also thank the advice and provision of plasmid pSBETa by Dr. Florence Vignols and Yves Meyer (University of Perpignan, France); the computer software helps by Ramón Nogales-Rangel and Alexis González-Policarpo; Eugenio Grau-Ferrando for kind advice and help for sequencing. This work was funded by the 10 month research contract MEC-FEDER to J.A.C.-M., 10 month research contract JAE-DOC to I.M.-S. and by the research project BIO2006-10138 from the MEC-FEDER of Spain to F.A.C.-M. In memoriam of Dr. Mari Cruz Cutanda-Pérez.

Open Access This article is distributed under the terms of the Creative Commons Attribution License which permits any use, distribution, and reproduction in any medium, provided the original author(s) and the source are credited.

\section{References}

Allen KN, Dunaway-Mariano D (2004) Phosphoryl group transfer: evolution of a catalytic scaffold. Trends Biochem Sci 29: 495-503

Altschul SF, Gish W, Miller W, Myers EW, Lipman DJ (1990) Basic local alignment search tool. J Mol Biol 215:403-410
Ames BN (1966) Assay of inorganic phosphate, total phosphate, and phosphatases. Methods Enzymol 8:115-118

Böhmer M, Schroeder JI (2011) Quantitative transcriptomic analysis of abscisic acid-induced and reactive oxygen species-dependent expression changes and proteomic profiling in Arabidopsis suspension cells. Plant J 67:105-118

Bradford MM (1976) A rapid and sensitive method for the quantization of microgram quantities of protein utilizing the principle of protein-dye binding. Anal Biochem 72:248-254

Burroughs AM, Allen KN, Dunaway-Mariano D, Aravind L (2006) Evolutionary genomics of the HAD superfamily: understanding the structural adaptations and catalytic diversity in a superfamily of phosphoesterases and allied enzymes. J Mol Biol 361:10031034

Caparrós-Martín JA, Reiland S, Köchert K, Cutanda MC, CuliáñezMacia FA (2007) Arabidopsis thaliana AtGpp 1 and AtGpp2: two novel low molecular weight phosphatases involved in plant glycerol metabolism. Plant Mol Biol 63:505-517

Collet JF, Stroobant V, Pirard M, Delpierre G, Van Schaftingen E (1998) A new class of phosphotransferases phosphorylated on an aspartate residue in an amino-terminal DXDX(T/V) motif. J Biol Chem 273:14107-14112

Corpet F, Servantm F, Gouzy J, Kahn D (2000) ProDom and ProDom-CG: tools for protein domain analysis and whole genome comparisons. Nucleic Acids Res 28:267-269

Cutanda MC (2003) Effect of altering levels of hexoses phosphate in carbohydrate metabolism and glucose signalling in yeast and plants. PhD thesis, Polytechnic University of Valencia, Valencia, Spain

Higgins D, Thompson J, Gibson T, Thompson JD, Higgins DG, Gibson TJ (1994) CLUSTAL W: improving the sensitivity of progressive multiple sequence alignment through sequence weighting, position-specific gap penalties and weight matrix choice. Nucleic Acids Res 22:4673-4680

Koonin EV, Tatusov RL (1994) Computer analysis of bacterial haloacid dehalogenases defines a large superfamily of hydrolases with diverse specificity. Application of an iterative approach to database search. J Mol Biol 244:125-132

Kupke T, Caparrós-Martín JA, Malquichagua Salazar KJ, CuliàñezMacià FA (2009) Biochemical and physiological characterization of Arabidopsis thaliana AtCoAse: a Nudix CoA hydrolyzing protein that improves plant development. Physiol Plant 135: 365-378

Kuznetsova E, Proudfoot M, Sanders SA, Reinking J, Savchenko A, Arrowsmith CH, Edwards AM, Yakunin AF (2005) Enzyme genomics: application of general enzymatic screens to discover new enzymes. FEMS Microbiol Rev 29:263-279

Kuznetsova E, Proudfoo M, Gonzalez CF, Brown G, Omelchenko MV, Borozan I, Carmel L, Wolf YI, Mori H, Savchenko AV, Arrowsmith CH, Koonin EV, Edwards AM, Yakunin AF (2006) Genome-wide analysis of substrate specificities of the Escherichia coli haloacid dehalogenase-like phosphatase family. J Biol Chem 281:36149-36161

Lahiri SD, Zhang G, Dai J, Dunaway-Mariano D, Allen KN (2004) Analysis of the substrate specificity loop of the HAD superfamily cap domain. Biochemistry 43:2812-2820

Lahiri SD, Zhang G, Dunaway-Mariano D, Allen KN (2006) Diversification of function in the haloacid dehalogenase enzyme superfamily: the role of the cap domain in hydrolytic phosphorus-carbon bond cleavage. Bioorganic Chem 34:394-409

Lambert C, Leonard N, De Bolle X, Depiereux E (2002) ESyPred3D: prediction of proteins 3D structures. Bioinformatics 18: $1250-1256$

Lu Z, Dunaway-Mariano D, Allen KN (2005) HAD superfamily phosphotransferase substrate diversification: structure and 
function analysis of HAD subclass IIB sugar phosphatase BT4131. Biochemistry 44:8684-8696

Lu Z, Dunaway-Mariano D, Allen KN (2008) The catalytic scaffold of the haloalkanoic acid dehalogenase enzyme superfamily acts as a mold for the trigonal bipyramidal transition state. Proc Natl Acad Sci USA 105:5687-5692

Maniatis T, Fritsch EF, Sambrook J (1982) Molecular cloning: a laboratory manual. Cold Spring Harbor Laboratory Press, Cold Spring Harbor

Morais MC, Zhang W, Baker AS, Zhang G, Dunaway-Mariano D, Allen KN (2000) The crystal structure of Bacillus cereus phosphonoacetaldehyde hydrolase: insight into catalysis of phosphorus bond cleavage and catalytic diversification within the HAD enzyme superfamily. Biochemistry 39:10385-10396

Morais MC, Zhang G, Zhang W, Olsen DB, Dunaway-Mariano D, Allen KN (2004) X-ray crystallographic and site-directed mutagenesis analysis of the mechanism of Schiff-base formation in phosphonoacetaldehyde hydrolase catalysis. J Biol Chem 279:9353-9361

Mueller WS, Hilbert B, Dueckershoff K, Roitsch T, Krischke M, Mueller MJ, Berger S (2008) General detoxification and stress responses are mediated by oxidized lipids through TGA transcription factors in Arabidopsis. Plant Cell 20:768-785

Murashige T, Skoog F (1962) A revised medium for rapid growth and bioassays with tobacco cultures. Physiol Plant 15:473-497

Norbeck J, Pahlman AK, Akhtar N, Blomberg A, Adler L (1996) Purification and characterization of two isoenzymes of DL-glycerol-3-phosphatase from Saccharomyces cerevisiae. Identification of the corresponding GPP1 and GPP2 genes and evidence for osmotic regulation of Gpp $2 p$ expression by the osmosensing mitogen-activated protein kinase signal transduction pathway. J Biol Chem 271:13875-13881

Rández-Gil F, Blasco A, Prieto JA, Sanz P (1995) DOGR1 and DOGR2: two genes from Saccharomyces cerevisiae that confer 2-deoxyglucose resistance when overexpressed. Yeast 11:1233-1240

Rao KN, Kumaran D, Seetharaman J, Bonanno JB, Burley SK, Swaminathan S (2006) Crystal structure of trehalose-6-phosphate phosphatase-related protein: biochemical and biological implications. Protein Sci 15:1735-1744

Schagger H, von Jagow G (1987) Tricine-sodium dodecyl sulfatepolyacrylamide gel electrophoresis for the separation of proteins in the range from 1 to $100 \mathrm{kDa}$. Anal Biochem 166:368-379
Schenk PM, Baumann S, Mattes R, Steinbiss HH (1995) Improved high-level expression system for eukaryotic genes in Escherichia coli using T7 RNA polymerase and rare ArgtRNAs. Biotechniques 19:196-200

Selengut JD (2001) MDP-1 is a new and distinct member of the haloacid dehalogenase family of aspartate-dependent phosphohydrolases. Biochemistry 40:12704-12711

Selengut JD, Levine RL (2000) MDP-1: a novel eukaryotic magnesium-dependent phosphatase. Biochemistry 39:8315-8324

Shin DH, Roberts A, Jancarik J, Yocota H, Kim R, Wemmer DE, Kim S-H (2003) Crystal structure of a phosphatase with a unique substrate binding domain from Thermotoga maritime. Protein Sci 12:1464-1472

Sussman I, Avron M (1981) Characterization and partial puri-fication of DL-glycerol-1-phosphatase from Dunaliella salina. Biochim Biophys Acta 661:199-204

The Arabidopsis Genome Initiative (2000) Analysis of the genome sequence of the flowering plant Arabidopsis thaliana. Nature 408:796-815

Tremblay LW, Dunaway-Mariano D, Allen KN (2006) Structure and activity analyses of Escherichia coli K-12 NagD provide insight into the evolution of biochemical function in the haloalkanoic acid dehalogenase superfamily. Biochemistry 45:1183-1193

Vicient CM, Delseny M (1999) Isolation of total RNA from Arabidopsis thaliana seeds. Anal Biochem 268:412-413

Wang W, Cho HS, Kim R, Jancarik J, Yokota H, Nguyen HH, Grigoriev IV, Wemmer DE, Kim S-H (2002) Structural characterization of the reaction pathway in phosphoserine phosphatase: crystallographic "snapshots" of intermediate states. J Mol Biol 319:421-431

Zhang G, Mazurkie AS, Dunaway-Mariano D, Allen KN (2002) Kinetic evidence for a substrate-induced fit in phosphonoacetaldehyde hydrolase catalysis. Biochemistry 41:13370-13377

Zhang G, Morais MC, Dai J, Zhang W, Dunaway-Mariano D, Allen KN (2004) Investigation of metal Ion binding in phosphonoacetaldehyde hydrolase identifies sequence markers for metal-activated enzymes of the HAD enzyme superfamily. Biochemistry 43: 4990-4997

Zimmermann P, Hirsch-Hoffmann M, Hennig L, Gruissem W (2004) GENEVESTIGATOR: Arabidopsis microarray database and analysis toolbox. Plant Physiol 136:2621-2632 